\title{
DISCLAIMER
}

This report was prepared as an account of work sponsored by an asency of the United States Government. Neither the United States Government nor any agency thereof, hor any of theit employees, makes any warranty, express or impiced, or an information, apparatus, product, or bility for the accuracy, completenees, or usefulneas of any inforinge privately owned rights. Referprocess disclosed, or represents that its use would not inf or service by trade name, trademark. ence herein to any specific commercill product, proces, or servimply its endorsement, recommanufacturer, or otherwive does not necessarily conentent or any asency thereof. The views mendation, or favoring by the United Slates Government or any asen state or reflect those of the and opinions of authon expreaced herein do not

\section{Solid Modeling Research at Lawrence Livermore National Laboratory: 1982-1985}

\author{
J. R. Kalibjian
}

Manuscript date: September 1, 1985

\section{LAWRENCE LIVERMORE NATIONAL LABORATORY University of California - Livermore, California - 94550}




\section{Contents}

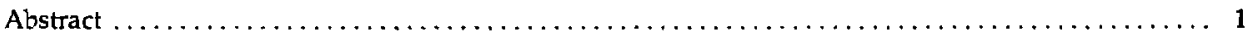

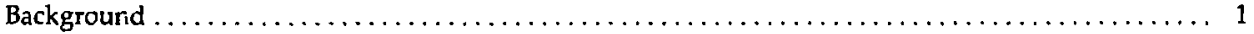

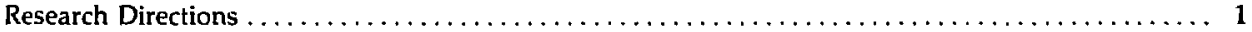

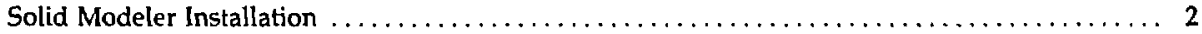

Extending TIPS-1 Geometric Coverage $\ldots \ldots \ldots \ldots \ldots \ldots \ldots \ldots \ldots \ldots \ldots \ldots \ldots \ldots \ldots, 2$

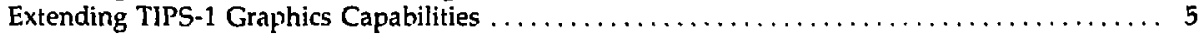

Improving Solid Modeler Performance at LLNL $\ldots \ldots \ldots \ldots \ldots \ldots \ldots \ldots \ldots \ldots \ldots \ldots \ldots$

Solid Modeler FEM Links $\ldots \ldots \ldots \ldots \ldots \ldots \ldots \ldots \ldots \ldots \ldots \ldots \ldots \ldots \ldots \ldots \ldots$

Programmatic Application of Solid Modeling at LLNL $\ldots \ldots \ldots \ldots \ldots \ldots \ldots \ldots \ldots \ldots \ldots \ldots \ldots, g$

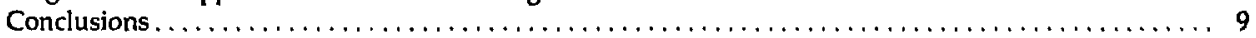

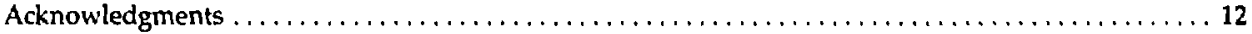

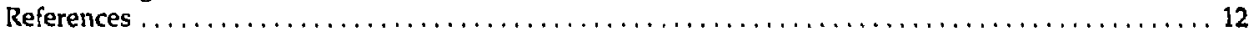




\title{
Solid Modeling Research at Lawrence Livermore National Laboratory: 1982-1985
}

\begin{abstract}
The Lawrence Livermore National Laboratory has sponsored solid modeling research for the past four years to assess this new technology and to determine its potential benefits to the Nuclear Weapons Complex. We summarize here the results of five projects implemented during our effort. First, we have installed two solid modeler codes, TIPS-1 (Technical Information Processing System-1) and PADL-2 (Part and Assembly Description Language), on the Laboratory's CRAY-1 computers. Further, we have extended the geometric coverage and have enhanced the graphics capabilities of the TIPS-1 modeler. To enhance solid modeler performance on our OCTOPUS computer system, we have also developed a method to permit future use of the Laboratory's network video system to provide high-resolution, shaded images at users' locations. Finally, we have begun to implement code that will link solid-modeler data bases to finite-element meshing codes.
\end{abstract}

\section{Background}

Automation of the design and manufacture of physical parts, known as Computer-Integrated Manufacturing, or CIM, has generated much interest at LLNL. Such automation promises to improve and speed communication between the agencies of the Nuclear Weapons Complex, which, in turn, will facilitate the production of better quality parts and reduce the overall design cycle time. Solid modeling ${ }^{1}$ provides the key to achieving CIM because solid modelers establish a mathematically complete part data base through which application programs can perform design analysis and manufacturing tasks. To acquire expertise in this area, the Mechanical Engineering
Department has funded solid modeling research at LLNL for the last four years. ${ }^{2-4}$ Further, to insure that the individuals involved in the effort would not work in a technical vacuum, the Laboratory began to spensor the work of two organizations involved in solid modeling and CIM research, specifically, the Production Automation Project at the University of Rochester, and the solid geometric modeling program at Computer Aided Manufacturing International, Inc. (CAM-I). The Laboratory believes that this unique position of research sponsorship and internal research will permit it to influence future solid modeling developments.

\section{Research Directions}

Although solid modeling is relatively new, the field already embraces many research areas. ${ }^{5}$ To use our resources most efficiently, we concentrated on those areas offering the greatest potential benefit to the Laboratory and to the Nuclear Weapons Complex. Specifically, we have examined:
- Installing solid modelers on supercomputers.

- Extending the geometric coverage of solid modelers.

- Enhancing the graphics capabilities of solid modeling systems. 
- Improving solid modeler performance on the LLNL OCTOPUS supercomputer network.

- Linking solid modeler data bases to existing LLNL finite element meshing (FEM) codes. The later two projects were added in the last year of the research effort.

\section{Solid Modeler Installation}

Before we started work on our other research areas, we had to obtain and install solid modeling systems on LLNL's OCTOPUS supercomputer network. These systems would serve as a framework upon which to test the algorithms we developed. Once we implemented some research ideas, we tested our improved modeling system on a programmatic application.

Only two solid modelers are readily available to research organizations at a reasonable cost. These modelers are PADL-2, produced by the University of Rochester's Production Automation Project, ${ }^{6}$ and TIPS- $1,{ }^{7}$ produced by Hokkaido University and available through CAM-I. PADL-2 is a multi-representational modeler-it supports constructive solid geometry (CSG), boundary file and octree representations. When a user inputs a part description into PADL-2, the CSG description is created first. The boundary file and octree descriptions are derived from the CSG representation when necessary. For example, if the user asks for a wireframe display, the boundary file is first created-or incrementally updated if the file already existed and some minor change was made-before graphical output is obtained. PADL-2 is an extremely rigorous modeler in that all intersections betw surfaces (faces) are expressly evaluated. This nelps implement a concept known as "neighborhoods," in which all surfaces that intersect at a particular edge are individuaily recorded. This feature promotes easy set memhership classification for two-point sets (e.g., for two point sets, say $A 1$ and $B 1, A 1$ is either in $B 1$, on $B 1$ or out of B1).

TIPS-1 is solely a CSG-like modeler. Unlike PADL-2, in which primitive solids are combined with the regularized set operators union, intersection, and difference, TIPS-1 has only one regularized set operator: union. Yet, difference and intersection operations can still be perform.ed by declaring primitive solids to be in "positive" or "negative" space, as shown in Figs. 1(a)-(d). TIPS-1 does not require that surface intersections be evaluated. Insiead, surface intersections are implied. This characteristic allowed us to easily add new geometric entities to the modeler.

Implementing TIPS-1 and PADL-2 on the Livermore Time Sharing System (LTSS) computer network, which contains three CDC 7600s, four CRAY-1s, and one CRAY XMP/48, was difficult due to the nonstandard features of the LTSS FORTRAN environment and the lack of documentation for the modelers themselves. We brought TIPS-1 up first and spent three man months installing it on the CRAYs. We directed most of our research effort toward TIPS-1 hecause we were only able to bring up the most recent version of PADL-2 on the CRAYs in January, 1985, after six man months of work.

\section{Extending TIPS-1 Geometric Coverage}

Once TIPS-1 was working on the CRAYs, we began to extend the code's geometric coverage. Our immediate goal was to incorporate the Wilson-Fowler spline into TIPS-1, In mathematics, a spline is a "smooth" curve that passes through specified data points [sometimes called knot points; see Fig. 2(a)]. A spline can be imagined as a thin piece of plastic or wood shaped by support structures. (At one time, this was how the contours of ships were laid out.) The many mathematical splines are differentiated by the form of the equation used to represent them, and many organizations use a particular spline tailored to the parts they design and manufacture-we use the Wilson-Fowler spline at Livermore.

In our modification, TIPS-1 accepts the knot points of the Wilson-Fowler spline, passes a smooth curve through them, and maps the curve into TIPS-1 $\mathrm{n}$, deling space. TIPS, 1 can then create a solid of rotation by sweeping the spine about an axis [Fig. 2(b)], or it can translate the 2-D surface along an arbitrary vector, thereby creating a solid of extrusion [Fig. 2(c)]. The top shown in Fig. 3 shows an actual example of an extruded and rotated Wilson-Fowler spline. The top was created by rotation, whereas its platform was created by an extrusion.

We performed additional work to extend the Wilson-Fowler spline to three-dimensional (3-D) space. We developed algorithms to create space curves that degenerated into Wilson-Fowler splines when projected into 2-D planes. Although the algorithms were tested, they were never actually added to the TIPS-1 code. 

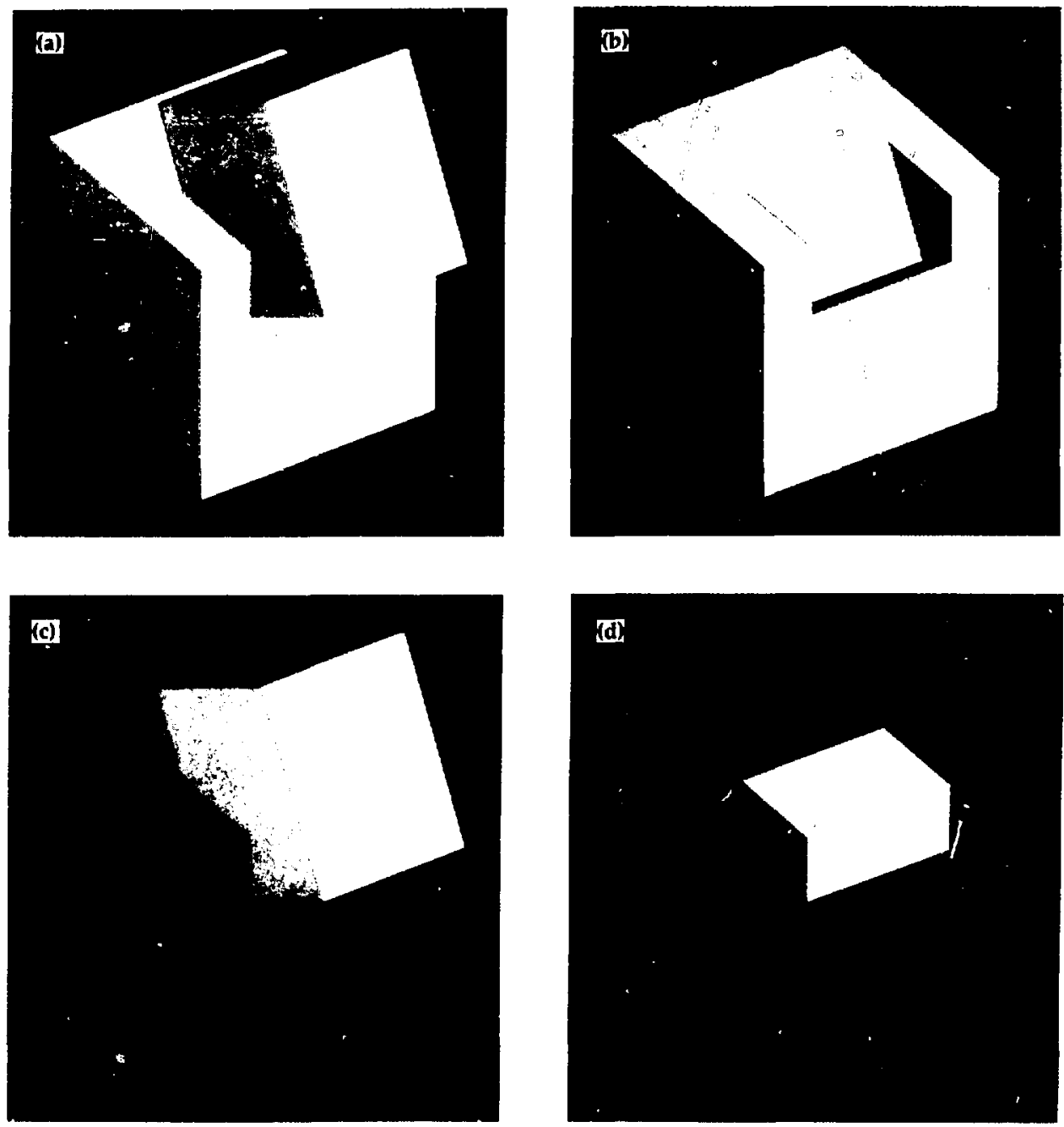

Figure 1. The different solids that can be defined by the union of two solid blocks by applying the concept of positive and negative space. (a) Both blocks in positive space (union). (b) Large block in positive space; small block in negative space (difference). (c) Small block in positive space; large block in negative space (difference). (d) Outside of large block in negative space; inside of small block in positive space (intersection).

Finally, we have also begun an effort to determine the relationship between Wilson-Fowler splines and other vendor-supported splines (e.g., B-splines, beta splines, and nu-splines). Along with this appraisal, we are also evaluating the op- timality of the Wilson-Fowler spline--compared to other splines-on typical Nuclear Weapons Complex (NWC) surfaces. Initial results indicate that the Wilson-Fowler spline is still the most appropriate spline for NWC needs. 

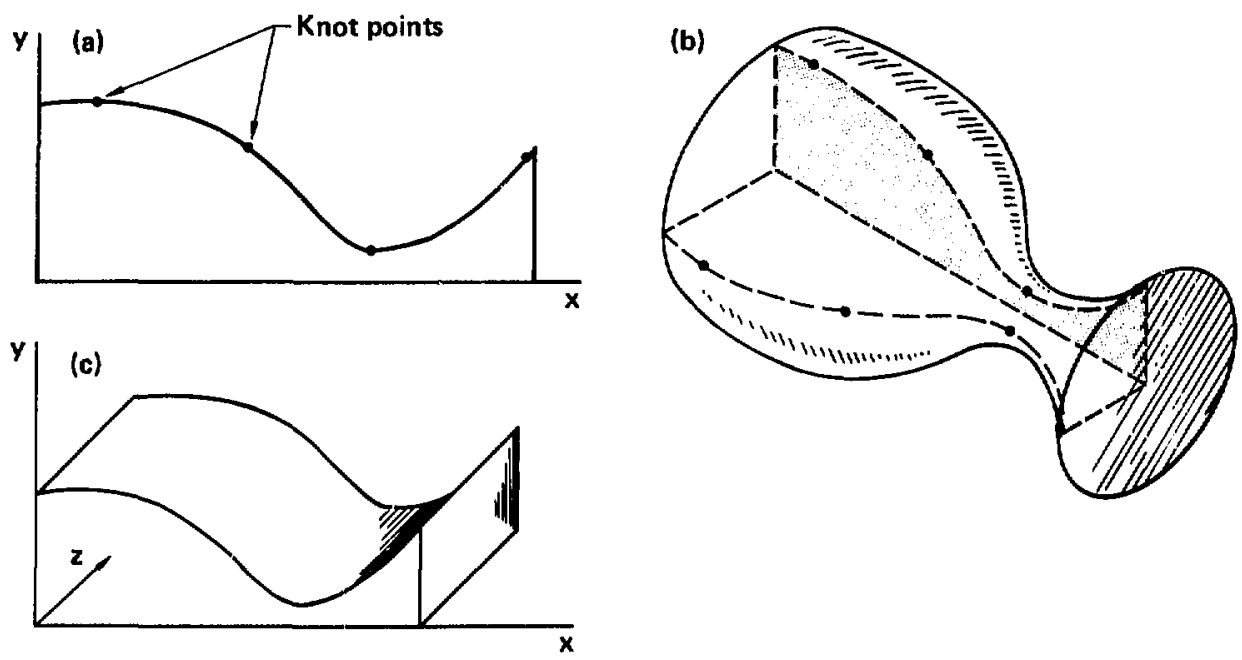

Figure 2. (a) Sample 2-D spline shown with knot points. (b) Solid of rotation generated by rotating plane defined in (a) about $x$ axis in 3-D space. (c) Solid of extrusion generated by translating the plane defined in (a) along the $z$ axis.

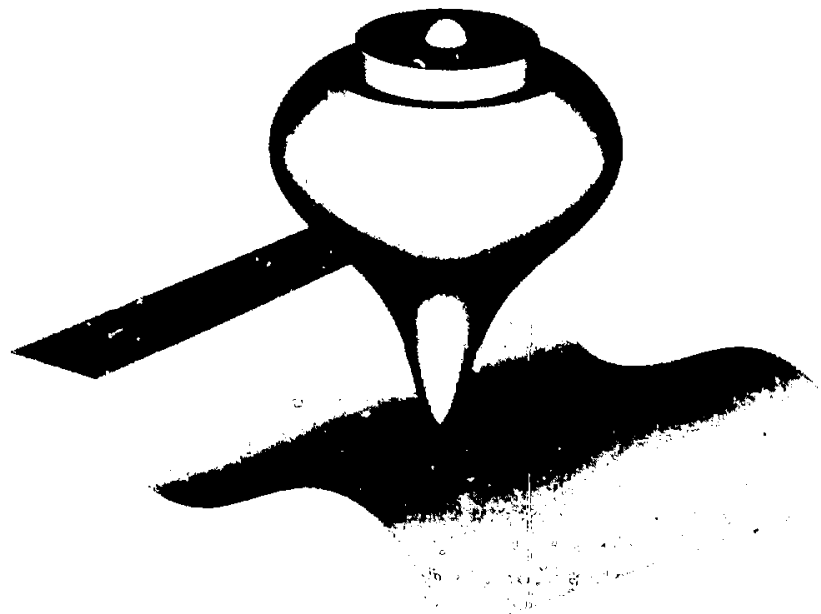

Figure 3. Solid surfaces generated by manipulating splines. (A spline is a smooth curve defi ed by a set of points; it may have a very complex shape.) The top was formed by rotating a spline about a vertical axis. The platform on which it stands was formed by sweeping a different spline along an arbitrary vector (extrusion). 


\section{Extending TIPS-1 Graphics Capabilities}

While extending the geometric coverage of the TIPS-1 solid modeler was an important priority, we also realized that such achievements are best documented by visual display of the new geometry. The original verrsion had limited graphics capabilities: only profile line images of an object could be generated. Immediately after we implemented TIPS. 1 on the CRAY-1 and before we did the Wilson-Fowler work, we wrote a hiddin-surface algorithm for TIPS-1 to obtain shadied pictures of defined objects (Fig. 4). Yet, this algorithm was not exact in approach. Later, after we completed the Wilson-Fowler work, we added an analytic ray-trace algorithm to TIPS-1.

\section{Ray Tracing in TIPS-1}

The analytic ray-trace algorithm ${ }^{y}$ can produce shacled images with shadows (Fig. 5), reflections (Fig 6), refractions, and transparencies (Fig. 7). The algorithm is conceptually simple. First, the viewer's eye position is established in viewer spare (Fig. 8). Next, a light source is positioned in

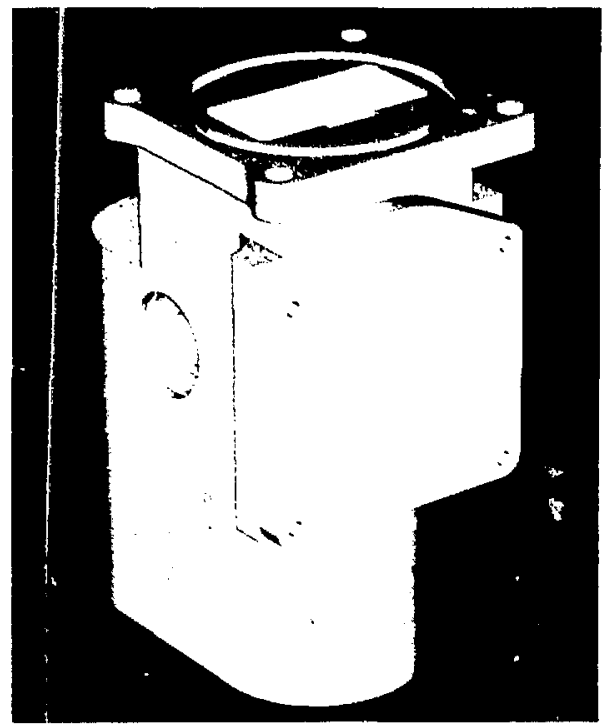

Figure 4. Cross section of an early example of the complexity and detail available from our solid modeling system with ray-tracing graphics.

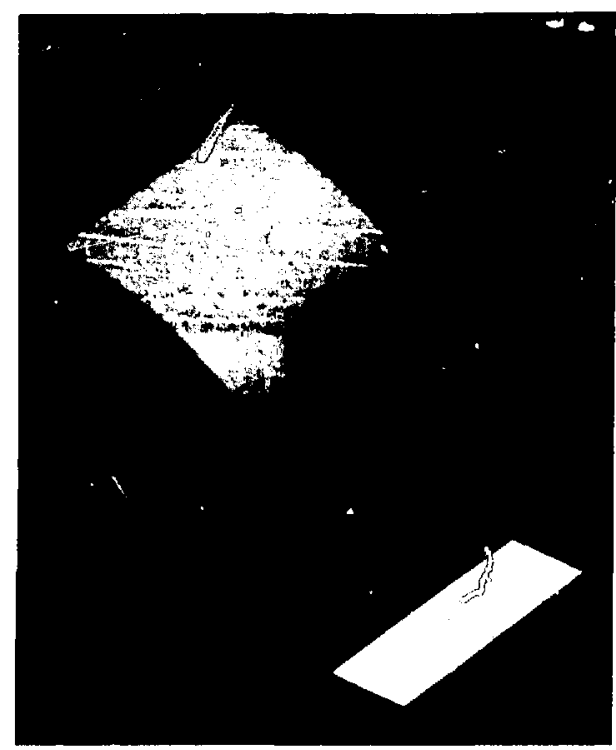

Figure 5. Shaded image of a TIPS-1-modeled part with shadows.

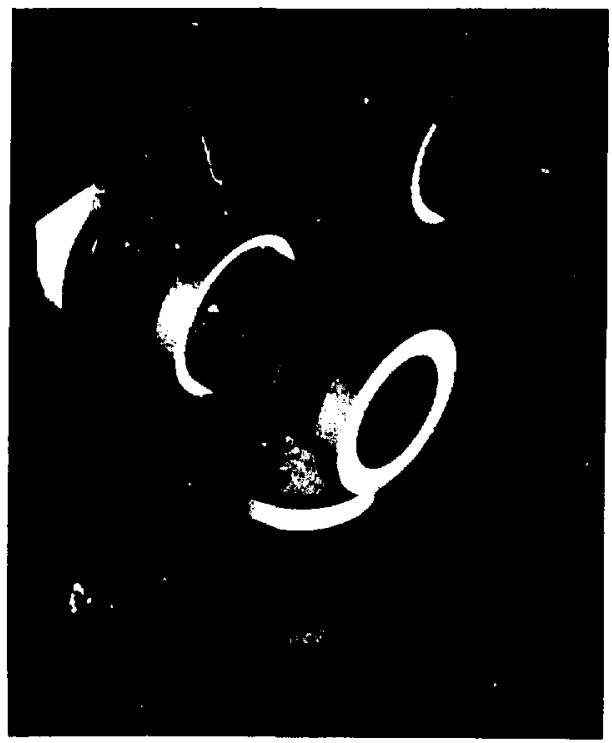

Figure 6. Shaded image of a TIPS-1-modeled part with reflections. 


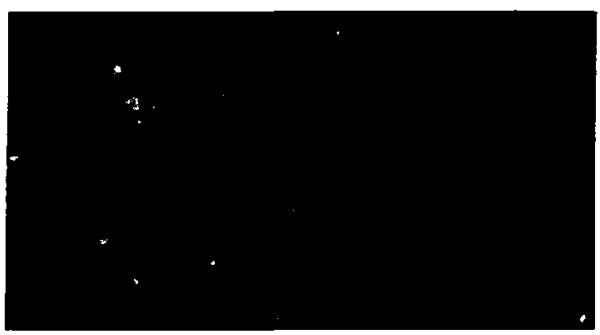

Figure 7. Shaded image of a TIPS-1-modeled part with refraction and transparency.

the object space, and a ray is cast from the eye position through a rectangular array of pixels into object space. The rectangular array represents the "film" on which the picture will be displayed. Once the ray is in object space, the point of intersection between the surface of the object and the ray is calculated, if one exists. If the ray intersects the object, the surface normal (a vector per- pendicular to the surface at the point of intersection) is calculated. The color intensity at the pixel will be proportional to the cosine between the surface normal and the vector to the light source. This algorithm was better than the first because it allowed the calculation of exact intursections between the ray and the surface; in this earlier algorithm these intersections were only approximated. Also, the algorithm allowed us to "follow" the ray after it made its first contact with the surface of the object, thus enabling us to produce reflections, shadows, refractions, and transparencies. Finally, to help produce more realistic images in our renderings, we integrated the Cook-Torrance? shading algorithm with our ray-casting code.

One drawback to the ray-casting process is the lirge amount of computer time needed to render an image. On average, 1-2 CRAY nimutes are needed for a typical picture. If the ray-casting process is to be made viable in a real-time modeling system, computation time must be reduced. Thus, we have begun to examine methods to speed up the rendering process. Basically, the goal is to reduce the number of rays cast at the object without

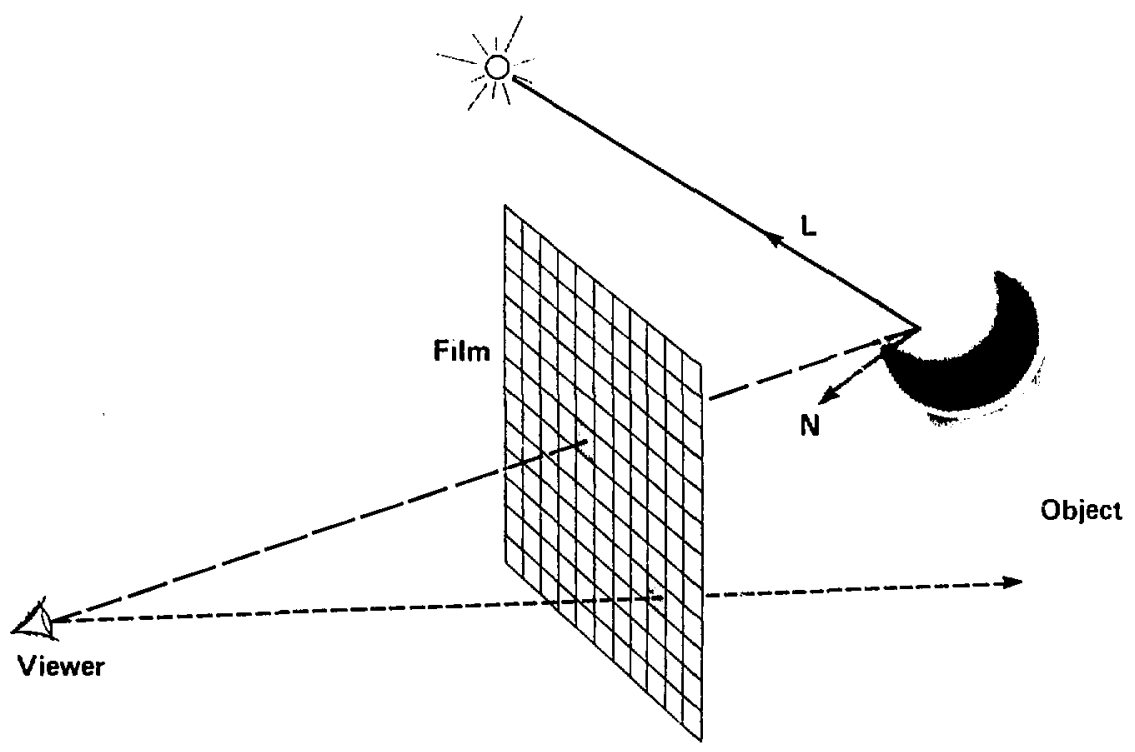

Figure 8. The ray-casting technique for specifying the color intensity of each pixel to produce a shaded display of a solid object. The algorithm projects a series of rays from a viewer's eye position, one for each pixel in a rectangular array, into the object space. For each ray that intersects the object, the algorithm calculates the color intensity required from the cosine of the angle between the surface normal $\mathbf{N}$ at that point and the illumination vector, $L$. 
losing any of its significant details. Our initial efforts have yielded a 25-percent decrease in the CRAY time needed to produce high-resolution pictures.

\section{TIPS-1 Scanline Algorithm}

To reduce rendering time further, we implemented a scanline algorithm in TIPS-1. The advantage of scanline algorithms is their speed; their disadvantages are that some of them use a great deal of memory and cannol render images with reflections, refractions, and shadows.

We implemented the z-buffer algorithm, which only renders objects made up of polygons. Thus, we first needed to implement routines that could take TIPS-1 analytical solid primitives, such as cones or cylindeis, in the CSG data base and turn them into polygons. Once the polygons are made, the object is rendered using the scanline algorithm. The shading effect produced by the algorithm is produced quite economically. The color intensities of the faces of the polygons are determined simply by interpolating the color intensities between their edges. The color intensity at the edges is determined by taking the cosine between the surface normal vector at the edge and the vector to the light source. So, for one polygon, only a very few surface normals must be calculated. This process directly contrasts with ray-tracing, in which a surface normal must he calculated for every point on the object.

The shading data, along with the depth of the polygon, are stored in the z-buffer and may be overwritten if a polygon with smaller z-values (corresponding to a polygon closer to the viewer) has the same position in the $x-y$ plane of a previously scan-converted polygon. Thus, hidden surface rendering is a by-product of the algorithm.

Initial results, for limited tests using blocks and cylinders in positive space only, indicate that:

- The scanline approach wil? obtain an order-of-magnitude speed-up over ray-casting only for objects consisting of many primitives.

- The ime required to "polygonize" the CSG data base is only a small fraction of the total elapsed time of the scanline rendering process for objects all in either positive or negative space.

Our next step will be to modify the code to make polygons from solids besides cones and blocks and to enable the code to render objects in both positive and negative space. A later project might involve implementing a hybrid rendering algorithm that uses elements of both scanline and ray-casting algorithms.

\section{Radiographs}

While the outward appearance of a part is important, the ability to examine its inside structure is equally vital. Inside structures are usually examined with $x$-ray pictures (radiographs). Such pictures reveal a density map of the object and may show flaws that could threaten its structural integrity. However, radiographs are difficult to interpret if the internal structure of the part is complex. Further, determining the placement of the $x$-ray source is also a non-trivial matter. Thus, it would be desirable to have a simulated radiograph of a flawless part to compare with the actual radiographs (since any discrepancies between the two pictures would be candidates for flaws) and to use as a tool to determine optimum placement of the $x$-ray source.

With some slight modifications to our analytic ray-trace algorithm, we are able to produce simulated radiographs ${ }^{10}$ of a modeled part [see Figs. 9(a)-(d)]. We believe this to be the first implementation of such a capability in a solid modeler. The key change in the algorithm involves allowing the ray, once it intersects the surface, to continue to propagate through the object. Subsequent intersections of the ray with other surfaces are recorded in a list until the ray exits the part. The list is then used to perform an integration of the solid material through which the ray passes. The color intensity of any pixel will be proportional to the amount of material through which the ray passes. We are still developing our radiograph production capability. Our next step will be to more exactly model the physics of the process.

\section{Improving Solid Modeler Performance at LLNL}

Simulated radiographs and shaded images of a part enable engineers to visually validate their designs. Engineers must be able to view these renderings quickly so as to minimize frustration with system interactivity and to enable them to keep their attention focused on the immediate problems at hand.

Unfortunately, the Livermore computer network has no real-time capability for viewing shaded images, although our CRAYs are able to produce the raster data at close to real-time speed. At present, only wireframe and low-resolution dithered images can be displayed off the CRAYs through our Television Monitor Display System 

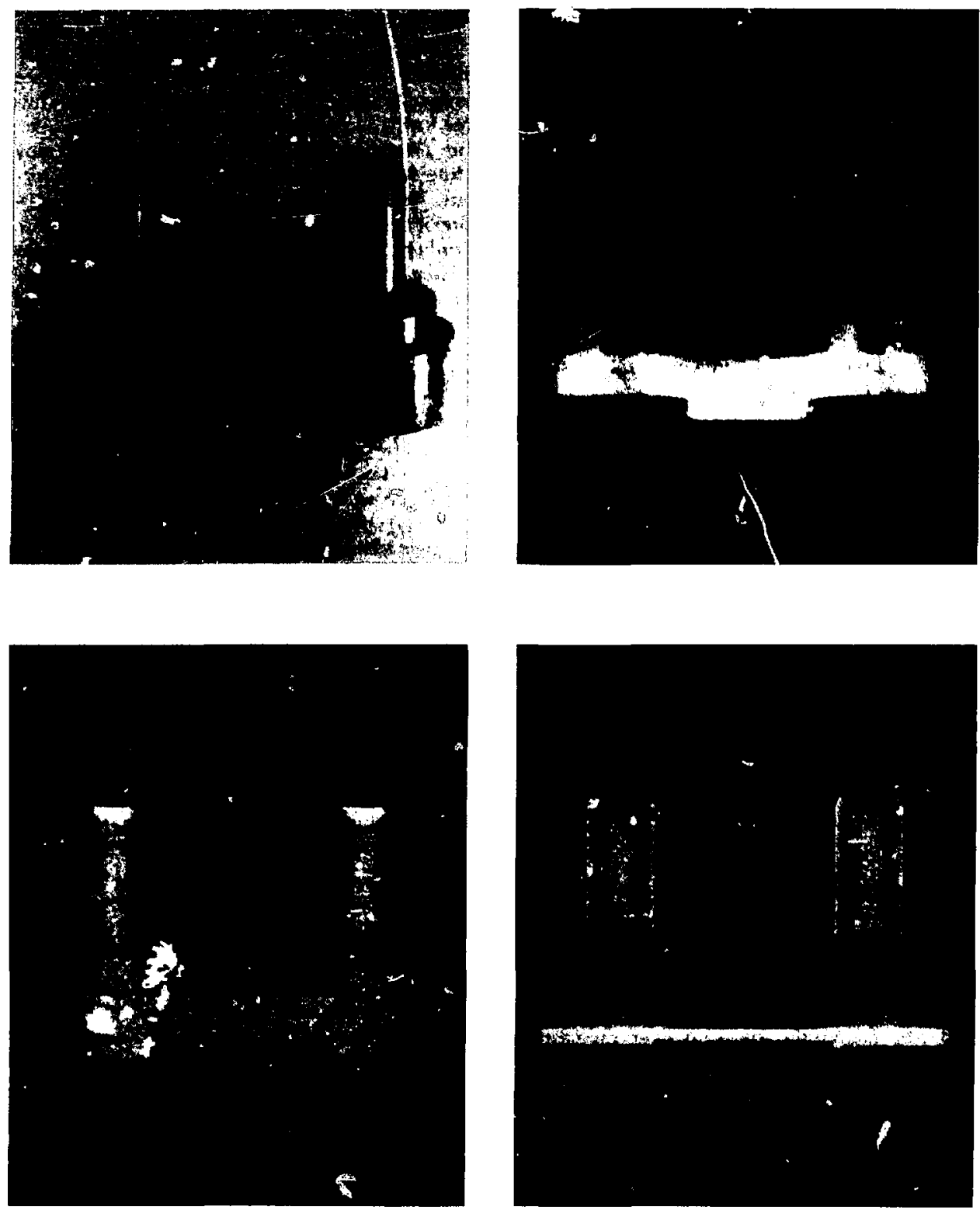

Figure 9. Radiograph of a modeled part. (a) Actual part. (b) Radiograph of actual part. (c) TIPS-1 shaded image of part. (d) TIPS-1 simulated $x$-ray image. Such a picture [shown in (d)] can be contrasted with the diagnostic radiograph of the real part [shown in (b)] to reveal flaws that might otherwise be undetected. In this case, no flaws were found. Simulation can also be used to find optimal positions of source, part, and $x$-ray film that give maximum flaw detection sensitivity. 
(TMDS). High-resolution color images can be obtained in a day's time with a Dicomed film recorder. Yet, if solid modeling is to become viable on our network, a method for quickly viewing high resolution shaded images in the office must exist. Using the network's existing video system (512- $\times 512-\times 1$-pixel, at $60-\mathrm{Hz}$ interlaced), we have devised a method to achieve this goal.

Basically, the existing video system will be used as a high-speed data channel. A shaded raster file will be sent over a TMDS cliannel $\left(2^{1 \mathrm{R}}\right.$ bits/TMDS frame) to the office, where a local, special-purpose frame grabber will ditize the data and make it available to a lacal frame buffer on an IBM PC. While the number of frames of data sent will depend on the picture resolution, 512- $\times$ 512- $\times$ 8-pixel pictures should be displayable in less than one minute. At this time, the frame grabber has been designed but not implemented.

\section{Solid Modeler FEM Links}

Although shaded images allow an engineer to qualitatively evaluate an object, finite element meshing is necessary for rigorous quantitative analysis of a part design. LLNL undoubtedly has developed the worId's best finite-element meshing (FEM) codes, e.g., MAZE, ${ }^{11}$ DYNA3-D, ${ }^{12}$ NIKE3-D, ${ }^{13}$ and INGRID. ${ }^{14}$ Yet, all suffer from a common drawback: the meshers themselves are not part of an integrated CAD/CAM solid modeling system; instead, they are stand-alone units. Thus, input of part descriptions is awkward; that is, a solid-model part description cannot be passed directly to the mesher-data must be manually input. This limitation implies that geametric validity of the part cannot be automatically assured and that the mesher will lose the opportunity to acquire a mathematically complete description of the part-information that it might he able to use for artificial intelligence approaches to mesh construction.

To alleviate this problem, we have beghun to implement code that will translate an input ASCII file written in the recently proposed ESP format ${ }^{15}$ to a MAZE input file (Fig. 10). ${ }^{16}$ We chose the ESP format because the specification will apparently be actopted by all vendors to permit part data exchange between different solid modelers. Although MAZE is a two-dimensional mesher, the objectives of the project are to develop a subset of core routines needed for the conversion process, and at the same time, to gain more familiarity with the ESP proposed standard. Once this is accomplished, the more difficult threedimensional case will be attempted.

\section{Programmatic Application of Solid Modeling at LLNL}

After we implemented the Wilson-Fowler spline in TIPS-1, we sought out a programmatic applicaiion in which to test our improved modeler. The results of our involvement were encouraging. Specifically, we found that:

- Drafters, accustomed to the traditional paper-and-pencil method, were quickly able to learn to use TIPS-1 effectively.
- TIPS-1 could model approximately 95 percent of the programmatic parts we attempted. We estimate that this figure would have been 40 to 50 percent without our TIPS-1 modifications.

- Our mass properties calculations on the $\mathbf{5 0}$ parts we modeled agreed to within one percent of known or otherwise calculated mass properties for those parts.

\section{Conclusions}

Our four-year effort has shown that it is possible to take a solid modeler off the shelf, to adapt it to the LLNL computer system, and to make the significant modifications necessary to handle our unique modeling needs. Thus, to a limited degree, we have established credibility in the solid mudeling area and may be able to influence some trend: in solid modeling. Yet, we have also determined that, while many applications for solid modeling exist at LLNL, no existing commercial solid modeler will meet all our needs-nor is one likely to for the next two or three years. Areas that still need work are:

- Automatic generation of process flans from a solid-model data base.

- Implementation of general surfaces swept along general space curves in a system where intersections are expressly evaluated. 
(a)

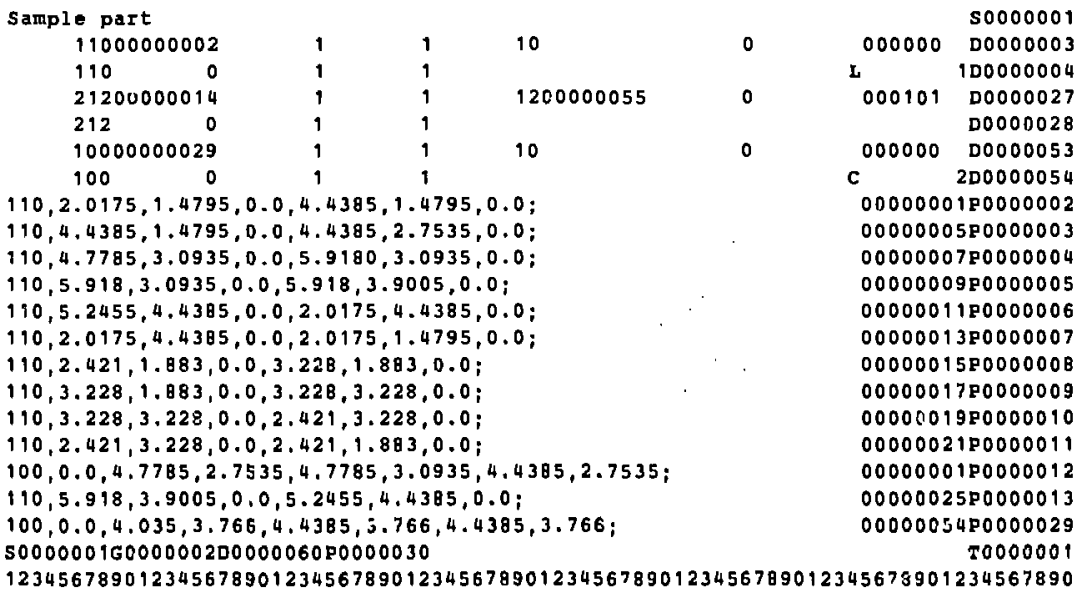

(b)

\begin{tabular}{|c|c|c|c|c|}
\hline \multicolumn{5}{|c|}{544} \\
\hline \multicolumn{5}{|c|}{$\begin{array}{l}544 \\
2104\end{array}$} \\
\hline $1 \mathrm{~d}$ & 1 & & & \\
\hline $1 \mathrm{p}$ & 22.0175 & 1.4795 & 4.4385 & 1.4795 \\
\hline $1 d$ & 2 & & & \\
\hline $1 p$ & 24.4385 & 1.4795 & 4.4335 & 2.7535 \\
\hline $1 d$ & 3 & & & \\
\hline $1 p$ & 24.7785 & 3.0935 & 5.9180 & 3.0935 \\
\hline $1 d$ & 4 & & & \\
\hline $1 p$ & 25.918 & 3.0935 & 5.918 & 3.9005 \\
\hline 10 & 5 & & & \\
\hline $1 p$ & 25.2455 & 4.4385 & 2.0175 & 4.4385 \\
\hline $1 d$ & 6 & & & \\
\hline $\operatorname{lp}$ & 22.0175 & 4.4385 & 2.0175 & 1.4795 \\
\hline 1d & 7 & & & \\
\hline $1 p$ & 22.421 & 1.883 & 3.228 & 1.883 \\
\hline 1d & 8 & & & \\
\hline lp & 23.228 & 1.883 & 3.228 & 3.228 \\
\hline ld & 9 & & & \\
\hline & 23.228 & 3.228 & 2.42 .1 & 3.228 \\
\hline ld & 10 & & & \\
\hline $\operatorname{lp}$ & 22.421 & 3.228 & 2.421 & 1.883 \\
\hline $1 d$ & 11 & & & \\
\hline $1 p$ & 14.4385 & 2.7535 & & \\
\hline $1 \mathrm{ap}$ & 4.7785 & 3.0935 & 4.7785 & 2.7535 \\
\hline $1 d$ & 12 & & & \\
\hline & 25.918 & 3.9005 & 5.2455 & 4.4385 \\
\hline 1d & 13 & & & \\
\hline $1 p$ & 14.4385 & 3.766 & & \\
\hline lad & 14.035 & 3.766 & 360 & \\
\hline $1 v$ & & & & \\
\hline tty & & & & \\
\hline
\end{tabular}

Figure 10. Initial test case of our ESP to MAZE translator. (a) Standard (IGES) part description file. (b) 2-D Mesher (MAZE) input commands derived by the translator from the input file. 


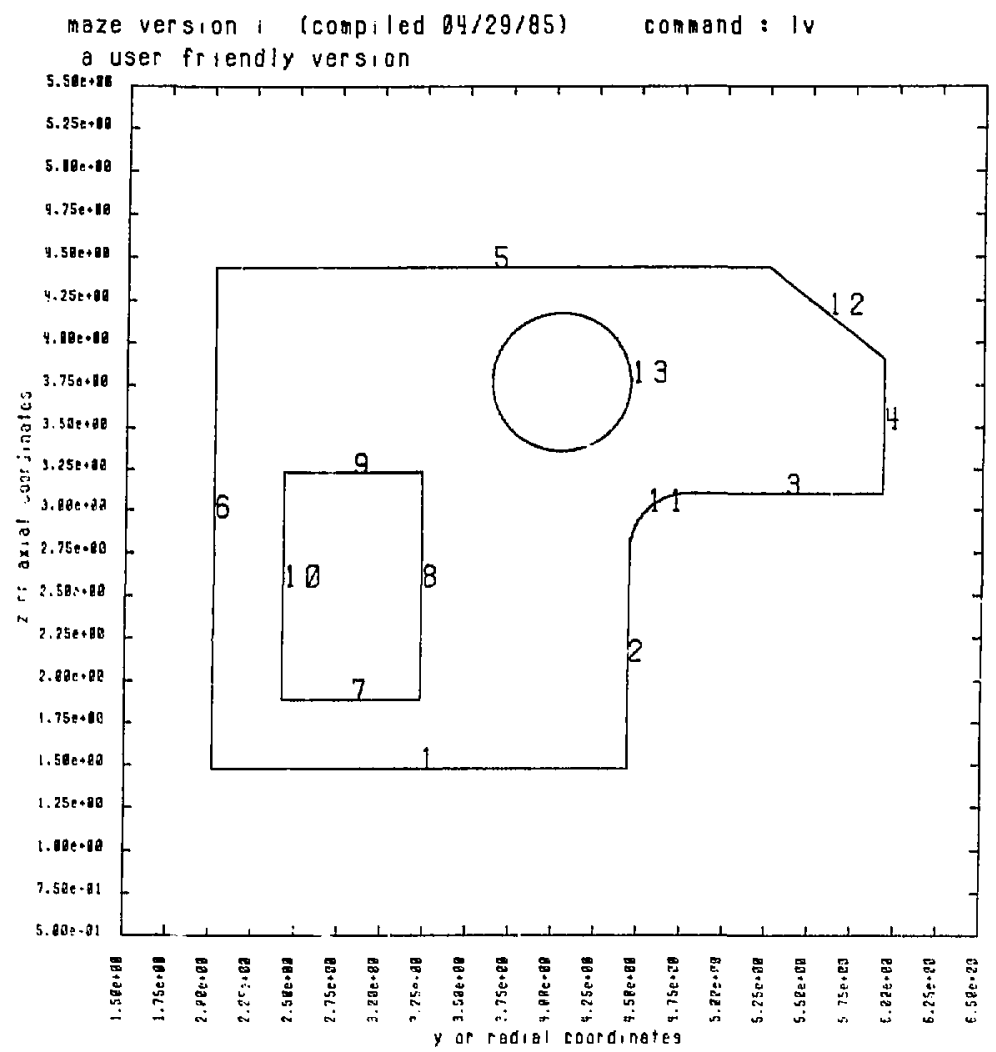

Figure 10(c). MAZE output resulting from the part description file of Fig. 10(a) and input commands of Fig. 10(b). 
- Automatic finite element mesh generation from a solid-model data base.

- Development of an accepted, useful, industry-wide, "neutral" data-exchange format for solid models.

- Implementation of full dimensioning and tolerancing capabilities in a solid modeler.

Our efforts will now be concentrated toward ensuring that future geometric modeling systems for different agencies of the Nuclear Weapons Complex are compatible. This compatibility will be achieved through the efforts of the recently formed Advanced Geometric Modeling Committee, which Livermore has been selected to head. While our primary concern is with interagency communication, we may atsu be able to examine unresolved research areas of consequence to the agencies involved. This activity will enable us to continually assimilate and evaluate solid modeling technology as it evolves, thus helping us to meet the present and future needs of LLNL and the Nuclear Weapons Complex.

\section{Acknowledgments}

Special thanks to M. K. Kong, G. W. Laguna, L. E. Taylor, and D. L. Vickers.

\section{References}

1. J. R. Kalibjian, Solid Modeling; Fonndation for Manufucturing Automation, Lawrence Livermure National Laboratory, Livermore, CA, UCRL-5365I (in progress).

2. H. Woo, Engineering Research Progress Semiannual Report-Firat Half FY-1985, Lawrence Livermore National Laboratory, Livermore, CA, UCID-19323-85-1 (in progress).

3. A. Goldberg, Mechanical Engintering Research Progiam Annual Report-FY-1983, Lawrence Livermore National Laboratory, Livermore, CA, UCID-19323-84-1 (1984), p. 81.

4. H. Woo, Engineerint Research Progress Report-FY-1984, Lawrence Livermore National Laboratory, Livermore, CA, UCID-19323-84-3 (1984), p. 69.

5. A. G. Requicha and H. B. Voelcker, "Solid Modeling: Current Status and Research Directions," Computer Graphics and Applications 3(7), 25 (1983).

6. C. Brown, "PADL-2: A Technical Summary," IEEE Computer Graphics and Applications 2(2), 69 (1982).

7. N. Okino, et al., "Theories for Graphics Processois in TIPS-1," Computers and Graplics 7(3-4), 243 (1983).

8. T. Whitted, "An Improved Illumination Model for Shaded Display," Communications of the ACM 23(6), 343 (1980).

9. R. Cook and K. Torrance, "A Reflectance Model for Computer Graphics," Computer Graphics 15(3), 307 (1981).

10. G. Laguna, Visunl Simulation of Radiographs, Lawrence Livermore National Laboratory, Livermore, CA, UCRL-53613 (1985).

11. J. O. Hallquist, MAZE: An Input Generator for DYNA2D and NIKE2D, Lawrence Livermore National Laboratory, Livermore, CA, UCID-19029, Rev. 1 (1982).

12. J. O. Hallquist, Theorefical Manual for DYNA3D, Lawrence Livermore National Laboratory, Livermore, CA, UCID-19401 (1983).

13. J. O. Hallquist, NIKE3D: An Implicit, Finite Deformation, Finite Element Code for Analyzing Static and Dynamic Response of Three-Dimensional Solids, Lawrence Livermore National Laboratory, Livermore, CA, UCID-18822 (1981).

14. D. W. Stillman, INGRID: A Three-Dimensional Finite Element Mesh Generator, Lawrence Livermore National Laboratory, Livermore, CA (in progress).

15. Advanced Geometry Comnittee, "E.S.P. Experimental Solids Proposal," (1984).

16. M. Kong, Implementation of â Translator for Data Exchange Between Contputer Aided Design Systemis and the MAZE Finite Element Meshing Program, Lawrence Livermore National Laboratory, Livermore, CA (in progress).

$\mathrm{JDH} / \mathrm{mc}$ 